\title{
A population-based study of intracranial arachnoid cysts: clinical and neuroimaging outcomes following surgical cyst decompression in children
}

\author{
Christian A. Helland, M.D., and Knut Wester, M.D., Ph.D. \\ Section for Neurosurgery, Department of Surgical Sciences, University of Bergen; and Department of \\ Neurosurgery, Haukeland University Hospital, Bergen, Norway
}

\begin{abstract}
Object. If, when, and how children with arachnoid cysts should undergo surgery has been a matter of debate. In the present study the authors describe long-term clinical and neuroimaging results in children with intracranial arachnoid cysts, treated in accordance with the authors' policy of performing surgery in most of these cases.

Methods. The study included 48 pediatric patients (age $<16$ years of age) who underwent surgery for treatment of an arachnoid cyst at Haukeland University Hospital between January 1987 and September 2004. Forty-one patients underwent cyst fenestration to the basal cisterns and posterior fossa. Long-term results were assessed retrospectively on the basis of medical and neuroimaging records. Additional information was obtained by means of a check-box questionnaire completed by the patients and their parents.

At their follow-up examinations, $82 \%$ of the patients had no or insignificant complaints, $14 \%$ reported no improvement, and $4 \%$ noted a worsening of symptoms. In $56 \%$ of the patients with appropriate imaging for analysis, the cyst was no longer visible on neuroimaging studies. In 23\%, the postoperative fluid volume was reduced to less than $50 \%$ of the original cyst volume, and in another $19 \%$, the volume was reduced but was larger than $50 \%$ of the original. The cyst was unchanged in only $2 \%$. There was a significant association between a volume reduction greater than $50 \%$ and clinical improvement. Three patients $(6 \%)$, all with temporal cysts, had minor complications that led to additional surgery in one patient. No complications caused permanent disability. Eight patients (17\%) underwent additional surgery due to suspected or demonstrated treatment failure.

Conclusions. Most children who underwent cyst fenestration via a craniotomy experienced a good long-term outcome with no severe complications.
\end{abstract}

\section{KEY WORDS • arachnoid cyst • decompression surgery - long-term follow up • pediatric neurosurgery}

$\mathrm{M}$ ODERN neuroimaging techniques have made the diagnosis of arachnoid cysts easier and have resulted in an increasing number of patients of all age groups presenting to neurosurgical departments with such cysts. Although children with arachnoid cysts may experience symptoms of intracranial hypertension or lateralizing symptoms, many present with only mild and rather unspecific symptoms such as headache, learning deficits, or behavioral disturbances. In infants, increasing head circumference may be the only indication. Nevertheless, in previous studies it has been documented that even cysts that cause only mild and unspecific symptoms may affect the function of neighboring cerebral tissue, ${ }^{7,36}$ causing impaired cognition, and that such cognitive deficits tend to normalize after surgery. ${ }^{5,16,35,42}$ We previously reported similar reversible cognitive deficits in adults. ${ }^{32,39,40}$

Abbreviations used in this paper: $\mathrm{COG}=$ clinical outcome group; $\mathrm{CP}=$ cystoperitoneal; GAT $1=$ glutaric aciduria type 1 ; ICD = International Classification of Diseases; $\mathrm{NOG}=$ neuroimaging outcome group; VP = ventriculoperitoneal.
In the majority of publications concerning symptomatic arachnoid cysts in children, the authors have advocated surgical intervention for these lesions. ${ }^{1,3,15,22,23,30,35}$ Nevertheless, some researchers favor a more conservative approach. ${ }^{31,37}$ Cysts in infants and older children have been reported to grow to a substantial size. ${ }^{21,41}$ Such observations would lend support to treatment by surgical decompression. ${ }^{21}$

Not only is it a matter of debate whether these children should undergo surgery, there are also diverging views on how such surgery should be performed. Some surgeons prefer fenestration through a craniotomy, ${ }^{8-10,23,27,33,35}$ whereas others advocate shunt insertion ${ }^{1,4,15,24,25,28}$ or fenestration through an endoscopic procedure..$^{3,29}$

By presenting the results from our department, it is our aim to contribute to the discussion of the questions raised earlier. On the basis of our increasing experience, we have gradually adopted more liberal indications regarding surgery for patients with arachnoid cysts. However, a policy of treating a relatively benign condition with surgery is justified only when a clear clinical benefit and a low risk of 
complications can be demonstrated. In the present study we describe clinical and neuroimaging outcomes in children who underwent surgery for an arachnoid cyst in our department between January 1987 and September 2004.

\section{Clinical Material and Methods}

\section{Hospital Structure and Study Population}

During the study period, Norway had a three-level, hierarchical hospital structure, in which local community hospitals served as primary referral centers. As a secondary referral center, most counties had a central county hospital with a pediatric department that could refer patients to one of the five university clinics, each serving a health region. Only university clinics have a neurosurgical department. A health region is a geographic area consisting of several counties with a well-defined population, and there is little or no overlap between these regions. Haukeland University Hospital is located on the southwestern coast of Norway; it serves three counties with a total population of 930,000 (as of 2003). During the study period, a hospital in the southern part of our health region referred some of its pediatric neurosurgical patients to another university hospital in Norway, thus reducing the population served at the time of the study to approximately 700,000 .

\section{Study Design and Data Extraction}

The present study is a questionnaire-based, retrospective study that includes all children (age $<16$ years) who underwent surgery for an arachnoid cyst at Haukeland University Hospital between January 1987 and September 2004. Patients with an arachnoid cyst were identified on the basis of information from the hospital's computer bank, and each patient with a diagnostic code of 348.0 (ICD 9) or G 93.0 (ICD 10) was registered in the study. Only patients with a verified arachnoid cyst were included. A cross-check was performed with hand-written operating room protocols from the time period as well as with typed surgical records kept by the senior author (K.W.) to ensure that all children who underwent surgery to treat an arachnoid cyst were included.

All the patients underwent a preoperative computed tomography or magnetic resonance imaging examination and a postoperative neuroimaging study within 72 hours. The patients were routinely readmitted 3 to 6 months after surgery for a postoperative visit that included clinical examination and a computed tomography or magnetic resonance imaging study. Patients in whom any postoperative abnormalities (for example, subdural hematoma or hygroma) appeared on neuroimages were followed up further until there was evidence of spontaneous remission or remission after surgical treatment of the complication.

The following data were extracted from patients' medical records: demographic information; presenting complaints; clinical findings; location and side of the cyst; Galassi type for the temporal cysts; ${ }^{11}$ postoperative change in cyst size; surgical method; and possible adverse effects of the operation identified by clinical or neuroimaging examination, such as subdural hygromas or hematomas, or new neurological symptoms or deficits.

During autumn 2004, a personal letter containing an explanation of the purpose of the investigation was sent to the parents or to the child if he or she was considered old enough. A check-box questionnaire, to be completed by the patient, the parents, or both, was enclosed together with a preaddressed, prestamped envelope. The questionnaire solicited information regarding postoperative changes in the presenting complaints, grading of the present level of complaints and level of function in everyday activities (shown on visual analog scales), any regrets parents or patients may have had about choosing a surgical option, and any new complaints that might have resulted from the surgery. There was also ample free space after each question for the respondents to give supplementary information.

\section{Patient Characteristics}

A total of 48 patients, 38 boys and 10 girls, were included in the study. The mean age of the patients at surgery was 6.3 years (range 1 month-15 years, median 5.7 years), and the mean follow-up duration was 84 months (range 6-180 months, median 90 months). The cysts were located in the temporal fossa in 29 patients (21 cysts on the left side, six on the right, and two bitemporal), overlying the frontal convexity in five patients (two cysts on the left side and three on the right), and in the posterior fossa in six patients (three cysts on the left side and three at the midline). Eight patients had cysts in other locations. The main preoperative complaints are summarized in Table 1.

In addition to the included patients, two boys underwent surgery for bitemporal arachnoid cysts. When children present with bitemporal cysts, there is a risk that they may have GAT 1, a congenital metabolic disorder that makes any kind of surgery or catabolic state a hazard to the patient. These two patients were found to have GAT 1 and were therefore not included in this study. Their cases have been described in detail elsewhere. ${ }^{26}$

\section{Surgical Procedures}

Forty-one patients $(85.4 \%)$ underwent primary surgery via craniotomy after induction of general anesthesia. During this operation, the parietal cyst membrane covering the inside of the dura mater was removed from its attachment to the surrounding cortex up to the tentorial slit in cases of middle fossa cysts. The arachnoid membrane that covered the brain surface (the visceral membrane) was not removed.

TABLE 1

Presenting symptoms in 48 children with arachnoid cysts

\begin{tabular}{lc}
\hline \hline \multicolumn{1}{c}{ Symptom } & No. of Patients $(\%)^{*}$ \\
\hline headache & $15(31)$ \\
impaired cognition & $13(27)$ \\
seizures & $10(21)$ \\
behavioral problems & $6(13)$ \\
increasing head circumference & $6(13)$ \\
cerebellar symptoms & $6(13)$ \\
motor symptoms & $5(10)$ \\
ocular symptoms & $4(8)$ \\
craniofacial anomaly & $3(6)$ \\
speech disorder (including dyslexia) & $3(6)$ \\
posttraumatic subdural hematoma $\dagger$ & $2(4)$ \\
\hline
\end{tabular}

* Some patients presented with more than one symptom.

$\dagger$ Cysts discovered by means of imaging investigation after head trauma. The patients had minimal symptoms from the cysts prior to injury. 
Any bridging veins coursing for some distance along the parietal membrane before they drained into the basal sinuses were coagulated and removed. This was done because we regularly observed oozing of blood from the site where these veins drained into the basal sinuses when manipulating the parietal membrane and the vein. Large bridging veins near the sphenoid ridge were not extirpated, as they coursed for only a very short distance along the membrane before draining and were believed to be supported by surrounding tissue in such a way that they would not move and cause bleeding. In cases of temporal cysts, the remaining medial membrane covering the basal structures (the tentorial slit, the oculomotor nerve, the carotid artery, and the optic nerve) was fenestrated, thus creating communication to the basal cisterns and the posterior fossa. When accessible, the arachnoid covering the sylvian fissure was also opened, thus creating communication to the subarachnoid space surrounding the carotid and middle cerebral arteries.

In three patients $(6.25 \%)$, an internal shunt was inserted from the cyst to the subdural space through a trephine hole after induction of local anesthesia. This method has been described in detail elsewhere. ${ }^{38}$ One patient received a cystoventricular shunt.

In two patients, primary surgery consisted of the insertion of a CP shunt. In one patient, a VP shunt was inserted as the primary measure to treat hydrocephalus. This patient's condition included multiple cerebral malformations in addition to an arachnoid cyst (dysmorphism of the cerebellum, posterior meningocele, bilateral schizencephaly, and a probable septooptic dysplasia).

\section{Scoring of Clinical Results}

The clinical results were categorized into one of four COGs based on information from medical reports and the questionnaire: in $\mathrm{COG} 1$, the preoperative complaints had disappeared entirely or were negligible; in COG 2, the preoperative complaints were clearly reduced but still present; in COG 3, the preoperative complaints were unchanged; and in $\mathrm{COG} 4$, the patient had more complaints after the operation.

\section{Scoring of Neuroimaging Results}

The neuroimaging results of the decompression, which are defined as the measured change in cyst volume on the neuroimaging follow-up study obtained after 3 to 6 months, were categorized into one of four possible NOGs: in NOG 1 , the cyst had disappeared and was no longer visible; in NOG 2, fluid volume amounting to less than $50 \%$ of the preoperative cyst volume was still visible at the site where the cyst had been; in NOG 3, fluid volume was also found, but the residual volume was greater than $50 \%$ of the preoperative cyst volume; and in NOG 4, no change in cyst volume could be observed. When the exact categorization was difficult to determine, the postoperative neuroimaging result was always assigned to the less favorable group.

\section{Answers to Letters}

Forty-eight letters with questionnaires were mailed to patients. Forty-four patients or parents (92\%) responded by filling out and returning the questionnaire.

\section{Statistical Analysis}

The statistical analyses were performed using SPSS (ver- sion 12.0 for Windows; SPSS Inc., Chicago, IL). An alpha level of 0.05 was used for all statistical calculations. Contingency tables were analyzed using Fisher exact tests or chisquare tests.

\section{Results}

\section{Clinical Outcomes}

The clinical results for different cyst locations are summarized in Table 2. Four patients did not respond and are therefore not included in the analyses of clinical results.

Twenty-one patients (48\%) were completely symptom free at their follow-up examination (COG 1). Another 15 patients $(34 \%)$ reported a significant reduction in the preoperative complaint (COG 2), whereas six patients (14\%) reported no reduction in their complaints (COG 3 ).

Two patients experienced a worsening in their symptoms after surgery (COG 4), despite an improvement shown on neuroimaging. Both had impaired cognition as the presenting complaint. One of these patients had a left temporal Galassi Type II cyst, and the other, a right frontal cyst. The frontal cyst was not visible on postoperative neuroimages (NOG 1), whereas the temporal cyst was reduced but was still greater than $50 \%$ of its original volume (NOG 3).

There was no difference in clinical outcome on the basis of cyst location. When all locations were considered, patients who underwent craniotomy and fenestration had a better outcome than those in whom shunts were placed ( $\mathrm{p}=$ 0.014; Fig. 1). Subgroup analyses for different cyst locations could not be performed because of the small numbers in the groups.

Clinical outcomes for the most common symptoms are summarized in Table 3. No difference was found in the comparison of clinical outcomes (COG 1 and COG 2 compared with COG 3 and COG 4) for the different symptoms.

\section{Neuroimaging Results}

The neuroimaging outcomes for different cyst locations are summarized in Table 4. Either pre- or postoperative imaging studies were missing in five patients, and these patients are therefore not included in the neuroimaging analysis. In $24(56 \%)$ of the remaining 43 patients, the cyst was no longer visible (NOG 1) on imaging studies. In another 10 patients $(23 \%)$, the postoperative fluid volume was reduced to less than $50 \%$ (NOG 2). In eight patients (19\%), the volume was reduced but was still larger than $50 \%$ of the preoperative cyst volume (NOG 3). In only one patient was there no change in postoperative volume (NOG 4).

No difference in neuroimaging outcomes was identified

TABLE 2

Clinical outcome in 44 patients with different cyst locations*

\begin{tabular}{lrrrl}
\hline \hline \multirow{2}{*}{$\begin{array}{c}\text { Cyst Location } \\
\text { (no. of patients) }\end{array}$} & \multicolumn{4}{c}{ No. of Patients (\%) } \\
\cline { 2 - 5 } & COG 1 & COG 2 & COG 3 & COG 4 \\
\hline temporal fossa (25) & $11(44)$ & $11(44)$ & $2(8)$ & $1(4)$ \\
frontal region (5) & $3(60)$ & $1(20)$ & $0(0)$ & $1(20)$ \\
posterior fossa (6) & $3(50)$ & $2(33)$ & $1(17)$ & $0(0)$ \\
other (8) & $4(50)$ & $1(13)$ & $3(37)$ & $0(0)$ \\
\hline
\end{tabular}

* Data were not available for four patients. 


\section{A. Helland and K. Wester}

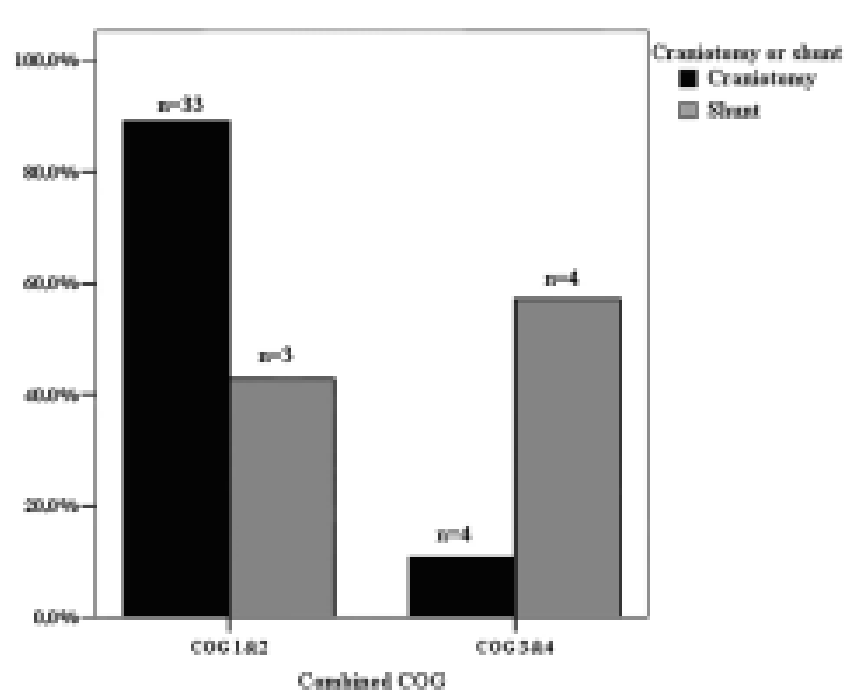

FIG. 1. Bar graph showing clinical outcome, expressed as the percentage of normal function, in children who underwent craniotomy fenestration compared with those in whom an internal shunt was placed. $\mathrm{n}=$ number of patients.

on the basis of cyst location or between the two most frequently used surgical techniques.

\section{Association Between Clinical and Neuroimaging Results}

In 42 patients, both the clinical and neuroimaging outcomes could be assessed. There was no strong association between postoperative volume and clinical outcome. However, after patients were dichotomized according to their good or poor clinical (COG 1 and 2 compared with COG 3 and 4) and neuroimaging (NOG 1 and 2 compared with NOG 3 and 4) outcomes, there was a significant association between a good clinical outcome and a good neuroimaging outcome $(\mathrm{p}=0.05)$.

Self-Reported Level of Function. A total of 28 patients $(67 \%)$ reported better function after surgery. Eight patients $(19 \%)$ reported no change, and six patients $(14 \%)$ reported a worse level of overall function after the operation. There was a slight discrepancy between the patients/parents' estimates of the postoperative functional level and the COG classification based only on the clinical complaints. Of the six patients who reported a worsening of overall function after surgery, two reported complete relief of the preoperative complaint (COG 1), two reported significant relief (COG 2), and two experienced a postoperative increase in the presenting complaint (COG 4). The symptoms of these

TABLE 3

Clinical outcome for all cyst locations stratified by the most common symptoms

\begin{tabular}{lllll}
\hline \hline & \multicolumn{4}{c}{ No. of Patients (\%)* } \\
\cline { 2 - 5 } \multicolumn{1}{c}{ Symptom } & COG 1 & COG 2 & COG 3 & COG 4 \\
\hline headache & $7(47)$ & $6(40)$ & $2(13)$ & $0(0)$ \\
impaired cognition & $3(23)$ & $6(46)$ & $2(15)$ & $2(15)$ \\
epilepsy† & $4(44)$ & $3(33)$ & $2(22)$ & $0(0)$ \\
\hline
\end{tabular}

* Some patients did not experience any of these symptoms.

$\dagger$ Follow-up data were missing in one patient.
TABLE 4

Neuroimaging outcome in 43 patients with different cyst locations*

\begin{tabular}{lrlllr}
\hline \hline & \multicolumn{4}{c}{ No. of Patients (\%) } \\
\cline { 2 - 4 } Cyst Location & NOG 1 & NOG 2 & NOG 3 & NOG 4 & Total \\
\hline temporal fossa & $13(54)$ & $5(21)$ & $6(25)$ & $0(0)$ & 24 \\
frontal region & $4(80)$ & $0(0)$ & $1(20)$ & $0(0)$ & 5 \\
posterior fossa & $2(33)$ & $3(50)$ & $0(0)$ & $1(17)$ & 6 \\
other & $5(63)$ & $2(25)$ & $1(12)$ & $0(0)$ & 8 \\
\hline
\end{tabular}

* Preoperative or postoperative imaging studies were missing in five patients.

patients were impaired cognition in two, behavioral disturbances in two, and epilepsy in one. The last of these children was diagnosed with an arachnoid cyst on the basis of increasing head circumference without any other clinical symptoms; in this child postoperative hydrocephalus developed and required insertion of a VP shunt. According to the postoperative neuroimaging results, three of the patients with worsening function were in NOG 1 , two were in NOG 2 , and one was in NOG 3.

\section{Complications and Treatment Failures}

Complications are defined in the following sections as additional pathological conditions caused by the treatment, whereas treatment failures are defined as a lack of effect of the treatment. None of the complications encountered were severe, nor did they lead to any permanent sequelae.

Complications. Three patients (6\%) experienced complications, all of whom had a temporal fossa cyst (Galassi Type II in two and bitemporal in one). The association between complications and cyst location was not significant. One patient had a subdural hygroma and one had a chronic subdural hematoma. In the third patient, who had bitemporal cysts, a postoperative hydrocephalus developed and was treated with a VP shunt. That patient was screened for GAT $1,{ }^{26}$ but this was not found. There was no difference in the complication rate between the different surgical techniques.

Treatment Failures. Eight patients (seven boys and one girl) underwent additional surgery because of suspected or demonstrated treatment failure (three temporal fossa, one posterior fossa, one suprasellar, and three in other locations). Four of these patients underwent fenestration through a craniotomy as their primary surgery; in the remaining four, a shunt (cystosubdural, CP, VP, and cystoventricular) was inserted as the primary procedure. Cyst location did not seem to influence the failure rate $(\mathrm{p}=0.132$ for temporal cysts compared with other locations); however, patients who had undergone craniotomy and fenestration as their primary surgery had a significantly lower risk of treatment failure than patients in whom a shunt was placed $(\mathrm{p}=0.01)$. Table 5 shows the relationship between the primary and subsequent procedures.

\section{Discussion}

In this report we show that most of this cohort of pediatric patients with an arachnoid cyst experienced clinical improvement after surgical cyst decompression as well as a significant postoperative reduction in complaints and a better level of overall function. Furthermore, this clinical ben- 
TABLE 5

Subsequent surgeries necessitated by treatment failure

\begin{tabular}{ccc}
\hline \hline \multirow{2}{*}{ Repeated Op Method } & \multicolumn{2}{c}{ Primary Op Method } \\
\cline { 2 - 3 } & Craniotomy & Shunt \\
\hline craniotomy (no. of patients) & 1 & $3 *$ \\
shunt (no. of patients) & $3 \dagger$ & 1 \\
revision of shunt & 1 & 3 \\
total no. of interventions & 5 & $8 *$ \\
\hline
\end{tabular}

* Two craniotomies were performed in one patient.

$\dagger$ A CP shunt in two patients (revised in one) and a cystosubdural shunt in one patient.

† A VP shunt for hydrocephalus required two revisions; an endoscopic third ventriculostomy was also required.

efit was accomplished without causing severe complications or additional disability.

The clinical improvement in our series seems to parallel the postoperative reduction in cyst volume, as children with the most pronounced volume reduction also experienced better clinical improvements. A similar clinical-neuroimaging correlation has been previously described by other authors, ${ }^{12,13,18,20,30}$ but it contrasts with our own findings in adults who underwent surgery to treat an arachnoid cyst (unpublished data), in whom clinical improvement seemed to be unrelated to the degree of volume reduction. This difference between adults and children is probably due to the fact that many of the children underwent surgery when their skulls were still growing. During childhood, the neurocranium is molded to fit its content. Thus, an arachnoid cyst creates surplus intracranial space by its presence during the growth of the skull, such as the enlarged middle fossa that can be seen in adults with temporal fossa cysts. In adults, this extra volume may in some cases be too large for the relieved brain to fill completely, even after successful cyst decompression, resulting in some residual fluid volume. In children, on the other hand, the still-growing brain may be able to fill in the vacant space left by the cyst. Surgical decompression at an early age may therefore prevent the mismatch between skull volume and brain volume that is often encountered after decompression in adults.

There is substantial evidence of critical developmental phases in childhood, during which specific abilities are learned and during which deprivation of stimuli that are important for the brain's development can cause permanent deficits. $2,6,14$ In several studies, arachnoid cysts have been shown to cause functional or metabolic deficits, or deficits in the perfusion of the underlying cerebral tissue. $7,17,32,35,36,39$, ${ }^{40,42}$ It can therefore be hypothesized that the presence of an arachnoid cyst during childhood may impair optimal brain development during critical developmental periods. Arachnoid cysts in children may therefore not only cause acute symptoms in some patients, but also affect the developing brain in more subtle ways, giving rise to learning difficulties and behavioral problems. A substantial number of patients in the present cohort were referred to our department because of behavioral problems. In our view, these possible impairments should be taken into account when considering surgical decompression of arachnoid cysts in children, because early decompression may relieve the pressure on the underlying cerebral tissue and thus allow more normal brain development.
The optimal treatment for arachnoid cysts in children has yet to be determined. The most common procedures-shunt placement and fenestration of the cyst-each have their benefits and drawbacks. ${ }^{1,4,8,10,15,19,23,24,30,33-35}$ Some authors advocate endoscopic techniques in the treatment of arachnoid cysts. ${ }^{3,29}$ In previous published comparisons of the results of fenestration and shunt insertion in the treatment of arachnoid cysts, the postoperative volume reduction seen on neuroimages has rarely been described accurately. In papers in which such information is given, a greater degree of volume reduction has been accredited to the shunt placement procedure. ${ }^{18}$ In our study, however, no difference in volume reduction was found between surgeries involving shunt placement and fenestration. Furthermore, clinical outcome was generally better in the patients who underwent fenestration, and the number of subsequent surgeries for treatment failures was significantly lower.

Cyst decompression through a fenestration also seems more physiologically sound than insertion of a $\mathrm{CP}$ shunt. A fenestration balances the pressure gradients that may exist between the cyst interior and the rest of the intracranial cavity, much in the same way as achieved by an endoscopic ventriculostomy. A CP shunt, on the other hand, creates a new intracranial pressure gradient, without equilibrating the intracystic and intracranial pressures. This has been reported to cause headache, which probably is related to the pressure inequality between the intracranial and the intracystic compartments. ${ }^{4}$

Although the numbers in our series are small, we consider the avoidance of shunt insertion in children a major treatment objective, and thus recommend fenestration through a craniotomy as the first treatment choice in children with arachnoid cysts.

\section{Conclusions}

Surgical decompression of arachnoid cysts in children yields good long-term outcomes in the majority of patients, with a low risk of complications or additional impairment. We believe that once the surgical option has been chosen, it should be performed as early as possible to avoid a skullbrain mismatch. An additional advantage of early surgery, at least in theory, is that it prevents pressure from the cyst on the underlying, developing brain. Fenestration of the cyst through a craniotomy seems the treatment of choice because of better outcome and a lower rate of treatment failure.

\section{References}

1. Arai H, Sato K, Wachi A, Okuda O, Takeda N: Arachnoid cysts of the middle cranial fossa: experience with 77 patients who were treated with cystoperitoneal shunting. Neurosurgery 39: 1108-1112, 1996

2. Blakemore C, Garey LJ, Vital-Durand F: The physiological effects of monocular deprivation and their reversal in the monkey's visual cortex. J Physiol 283:223-262, 1978

3. Choi JU, Kim DS, Huh R: Endoscopic approach to arachnoid cyst. Childs Nerv Syst 15:285-291, 1999

4. Ciricillo SF, Cogen PH, Harsh GR, Edwards MS: Intracranial arachnoid cysts in children. A comparison of the effects of fenestration and shunting. J Neurosurg 74:230-235, 1991

5. Colameco S, DiTomasso RA: Arachnoid cyst associated with psychological disturbance. J Med Soc N J 79:209-210, 1982 
6. Daw NW, Wyatt HJ: Kittens reared in a unidirectional environment: evidence for a critical period. J Physiol 257:155-170, 1976

7. De Volder AG, Michel C, Thauvoy C, Willems G, Ferriere G: Brain glucose utilisation in acquired childhood aphasia associated with a sylvian arachnoid cyst: recovery after shunting as demonstrated by PET. J Neurol Neurosurg Psychiatry 57: 296-300, 1994

8. Di Rocco C, Tamburrini G: Shunt dependency in shunted arachnoid cyst: a reason to avoid shunting. Pediatr Neurosurg 38:164, 2003

9. Fewel ME, Levy ML, McComb JG: Surgical treatment of 95 children with 102 intracranial arachnoid cysts. Pediatr Neurosurg 25:165-173, 1996

10. Galassi E, Gaist G, Giuliani G, Pozzati E: Arachnoid cysts of the middle cranial fossa: experience with 77 cases treated surgically. Acta Neurochir Suppl (Wien) 42:201-204, 1988

11. Galassi E, Tognetti F, Gaist G, Fagioli L, Frank F, Frank G: CT scan and metrizamide CT cisternography in arachnoid cysts of the middle cranial fossa: classification and pathophysiological aspects. Surg Neurol 17:363-369, 1982

12. Garcia-Bach $M$, Isamat F, Vila F: Intracranial arachnoid cysts in adults. Acta Neurochir Suppl (Wien) 42:205-209, 1988

13. Geissinger JD, Kohler WC, Robinson BW, Davis FM: Arachnoid cysts of the middle cranial fossa: surgical considerations. Surg Neurol 10:27-33, 1978

14. Grossman ML: Early child development in the context of mothering experiences. Child Psychiatry Hum Dev 5:216-223, 1975

15. Harsh GR IV, Edwards MSB, Wilson CB: Intracranial arachnoid cysts in children. J Neurosurg 64:835-842, 1986

16. Horiguchi T, Takeshita K: Cognitive function and language of a child with an arachnoid cyst in the left frontal fossa. World J Biol Psychiatry 1:159-163, 2000

17. Hund-Georgiadis M, Yves Von Cramon D, Kruggel F, Preul C: Do quiescent arachnoid cysts alter CNS functional organization? A fMRI and morphometric study. Neurology 59:1935-1939, 2002

18. Kandenwein JA, Richter HP, Börm W: Surgical therapy of symptomatic arachnoid cysts-an outcome analysis. Acta Neurochir (Wien) 146: 1317-1322, 2004

19. Kang JK, Lee KS, Lee IW, Jeun SS, Son BC, Jung CK, et al: Shunt-independent surgical treatment of middle cranial fossa arachnoid cysts in children. Childs Nerv Syst 16:111-116, 2000

20. Koch CA, Moore JL, Voth D: Arachnoid cysts: how do postsurgical cyst size and seizure outcome correlate? Neurosurg Rev 21:14-22, 1998

21. Kumagai M, Sakai N, Yamada H, Shinoda J, Nakashima T, Iwama T, et al: Postnatal development and enlargement of primary middle cranial fossa arachnoid cyst recognized on repeat CT scans. Childs Nerv Syst 2:211-215, 1986

22. Levy ML, Meltzer HS, Hughes S, Aryan HE, Yoo K, Amar AP: Hydrocephalus in children with middle fossa arachnoid cysts. J Neurosurg 101 (2 Suppl Pediatrics):25-31, 2004

23. Levy ML, Wang M, Aryan HE, Yoo K, Meltzer H: Microsurgical keyhole approach for middle fossa arachnoid cyst fenestration. Neurosurgery 53:1138-1145, 2003

24. Locatelli D, Bonfanti N, Sfogliarini R, Gajno TM, Pezzotta S: Arachnoid cysts: diagnosis and treatment. Childs Nerv Syst 3: 121-124, 1987

25. Lodrini S, Lasio G, Fornari M, Miglivacca F: Treatment of supratentorial primary arachnoid cysts. Acta Neurochir (Wien) 76: $105-110,1985$

26. Lütcherath V, Waaler PE, Jellum E, Wester K: Children with bilateral temporal arachnoid cysts may have glutaric aciduria type 1 (GAT1); operation without knowing that may be harmful. Acta Neurochir (Wien) 142:1025-1030, 2000

27. Marinov M, Undjian S, Wetzka P: An evaluation of the surgical treatment of intracranial arachnoid cysts in children. Childs Nerv Syst 5:177-183, 1989

28. McBride LA, Winston KR, Freeman JE: Cystoventricular shunting of intracranial arachnoid cysts. Pediatr Neurosurg 39:323-329, 2003

29. Nowoslawska E, Polis L, Kaniewska D, Mikolajczyk W, Krawczyk J, Szymanski W, et al: [A comparison of neuro-endoscopic techniques with other surgical procedures in the treatment of arachnoid cysts in children.] Neurol Neurochir Pol 37: 587-600, 2003 (Pol)

30. Oberbauer RW, Haase J, Pucher R: Arachnoid cysts in children: a European co-operative study. Childs Nerv Syst 8:281-286, 1992

31. Parsch CS, Krauss J, Hofmann E, Meixensberger J, Roosen K: Arachnoid cysts associated with subdural hematomas and hygromas: analysis of 16 cases, long-term follow-up, and review of the literature. Neurosurgery 40:483-490, 1997

32. Raeder MB, Helland CA, Hugdahl K, Wester K: Arachnoid cysts cause cognitive deficits that improve after surgery. Neurology 64:160-162, 2005

33. Raffel C, McComb JG: To shunt or to fenestrate: which is the best surgical treatment for arachnoid cysts in pediatric patients? Neurosurgery 23:338-342, 1988

34. Richard KE, Dahl K, Sanker P: Long-term follow-up of children and juveniles with arachnoid cysts. Childs Nerv Syst 5: 184-187, 1989

35. Sato H, Sato N, Katayama S, Tamaki N, Matsumoto S: Effective shunt-independent treatment for primary middle fossa arachnoid cyst. Childs Nerv Syst 7:375-381, 1991

36. Sgouros S, Chapman S: Congenital middle fossa arachnoid cysts may cause global brain ischaemia: a study with 99Tc-hexamethylpropyleneamineoxime single photon emission computerised tomography scans. Pediatr Neurosurg 35:188-194, 2001

37. Sommer IEC, Smit LME: Congenital supratentorial arachnoidal and giant cysts in children: a clinical study with arguments for a conservative approach. Childs Nerv Syst 13:8-12, 1997

38. Wester K: Arachnoid cysts in adults: experience with internal shunts to the subdural compartment. Surg Neurol 45:15-23, 1996

39. Wester K, Hugdahl K: Arachnoid cysts of the left temporal fossa: impaired preoperative cognition and postoperative improvement. J Neurol Neurosurg Psychiatry 59:293-298, 1995

40. Wester K, Hugdahl K: Verbal laterality and handedness in patients with intracranial arachnoid cysts. J Neurol 250:36-41, 2003

41. Wester K, Moen G: Documented growth of a temporal arachnoid cyst. J Neurol Neurosurg Psychiatry 69:699-700, 2000

42. Zaatreh MM, Bates ER, Hooper SR, Palmer G, Elmenshawi EE, Courvoisie HE, et al: Morphometric and neuropsychologic studies in children with arachnoid cysts. Pediatr Neurol 26: 134-138, 2002

Manuscript received April 7, 2006.

Accepted in final form August 16, 2006.

This work was supported by a grant to Dr. Helland from the Helse Vest Regional Research Fund 2004 (Project No. 911065).

Address reprint requests to: Knut Wester, M.D., Ph.D., Department of Neurosurgery, Haukeland University Hospital, N-5021 Bergen, Norway. email: kgwe@ helse-bergen.no. 